\title{
The first cross-calibration of Imaging Atmospheric Cherenkov Telescopes with a UAV-based airborne calibration platform
}

\author{
Jacques Muller, ${ }^{a, *}$ Anthony M. Brown ${ }^{b}$ and Mathieu de Naurois ${ }^{a}$ \\ ${ }^{a}$ Laboratoire Leprince-Ringuet, CNRS/IN2P3, Ecole Polytechnique, Institut Polytechnique de Paris, \\ Palaiseau, France \\ ${ }^{b}$ Centre for Advanced Instrumentation, Department of Physics, Durham University, \\ Durham, UK DHI 3LE, United Kingdom \\ E-mail: jacques.muller@polytechnique.edu
}

The Cherenkov Telescope Array (CTA) aims to have unprecedented accuracy and sensitivity, affording us the ability to understand the mysteries of the high energy universe. This unprecedented accuracy forces us to improve current calibration procedures, or indeed pioneer new techniques, to ensure the envisaged CTA performance. CTA will infer the energy of the gamma-rays it detects from the amount of Cherenkov radiation it observes. As such, the optical efficiency of the telescopes needs to be monitored, and its wavelength dependent degradation, which might be different for different telescope types, needs to be determined. Based on the results of a feasibility study, a novel cross-calibration method with an unmanned aerial vehicle (UAV) was tested on the H.E.S.S. telescope array, leading to the first cross-calibration of an Imaging Atmospheric Cherenkov Telescope (IACT) array with a single light source. In this talk, we present the crosscalibration results from a first test campaign in which we determine the relative optical efficiencies of the four HESS-I telescopes by successfully recording light from the UAV-mounted nanosecond pulsed UV light source simultaneously in all four telescopes. In addition, we show that the UAV data can be used to monitor the pointing accuracy at the level of at least tens of arcseconds and we give an outlook on other potential use cases of UAVs such as the monitoring of the atmospheric state.

$37^{\text {th }}$ International Cosmic Ray Conference (ICRC 2021)

July 12th - 23rd, 2021

Online - Berlin, Germany

\footnotetext{
${ }^{*}$ Presenter
} 


\section{Introduction}

The next generation Imaging Atmospheric Cherenkov Telescope (IACT) array, the Cherenkov Telescope Array (CTA), will have about an order of magnitude improvement in sensitivity and an unprecedented angular and energy resolution [1]. This improvement in performance will be reached using three telescope classes optimised to observe different photon energies and an envisaged total of more than 100 telescopes on two sites, one in the Northern hemisphere, and the other in the Southern hemisphere. This introduces new calibration challenges: the wavelength dependent characterisation of the optical efficiencies of the individual telescopes and its evolution with time. Advances in unmanned aerial vehicle (UAV) technology have made UAVs an attractive possibility for calibrating astronomical facilities [2-6], and so the possibility of cross-calibrating the optical efficiencies of the individual telescopes of a Cherenkov telescope array has been investigated in a feasibility study [6].

This novel method has been tested on the largest IACT array of current generation, the H.E.S.S. array in the Khomas Highlands in Namibia at an altitude of $1800 \mathrm{~m}$, in May 2018. H.E.S.S. is constituted of five telescopes, four with a dish diameter of $12 \mathrm{~m}$ (HESS-I telescopes, CT1-4), and one with a dish diameter of $28 \mathrm{~m}$ (HESS-II telescope, CT5). Due to operational constraints, CT5 was not yet part of this first proof of concept. The prototype used in this first test-campaign consisted of a commercially available octocopter equiped with an avionics suite consisting of gyroscopes, accelerometers and a Global Navigation Satellite System (GNSS) receiver on which a custom-built LED-based pulsed light source was mounted. It was able to produce $4 \mathrm{~ns}$ duration flashes at a wavelength of $400 \mathrm{~nm}$ across a large intensity range [7]. In this first campaign, it was triggered at a frequency of $1 \mathrm{~Hz}$ and fired through a $50^{\circ}$ circular top-hat diffuser.

Ultimately, it is planned for the UAV to fly a vertical pattern from the centre of the array to implement the UAV-based calibration technique [6], however for this test campaign, the UAV took off at the H.E.S.S. residence about $800 \mathrm{~m}$ south-east of the H.E.S.S. array and went up to altitudes of $200 \mathrm{~m}$, thus staying at low elevation as viewed from the telescopes. This allows us to achieve a distance of about $820 \mathrm{~m}$ between the UAV and the telescopes without a long climbing time and so to maximise the time at the calibration altitude, and to reduce the requirement of light control at the take-off location compared to a take-off location closer to the telescopes. For each calibration run, the four HESS-I telescopes were pointed to the nominal position of the UAV in convergent pointing mode ${ }^{1}$ and the UAV was flown to this position. Standard observation trigger settings were used with the exception that events triggering only one telescope were accepted in order to record enough cosmic events to do a proper pedestal calibration. Since the telescopes are focused to observe atmospheric showers at a distance of $8 \mathrm{~km}$ above the H.E.S.S. array [8], the light from the UAV, at a distance of $800 \mathrm{~m}$ is smeared across multiple pixels.

Two successful UAV calibration runs were taken under a very similar configuration for each of which the UAV was visible to the telescopes for $400 \mathrm{~s}$ to $500 \mathrm{~s}$. The first, run A, was taken on the $20^{\text {th }}$ of May 2018 at 21:29 UTC, and recorded a total of 41000 events per telescope (signal and background combined). The second, run B, was taken on the $21^{\text {st }}$ of May 2018 at 22:33 UTC, and recorded a total of 27000 events per telescope.

${ }^{1}$ Convergent pointing mode means that the telescopes are pointing towards a fixed position (here the nominal position of the UAV) instead of pointing parallel to a position on the sky. 

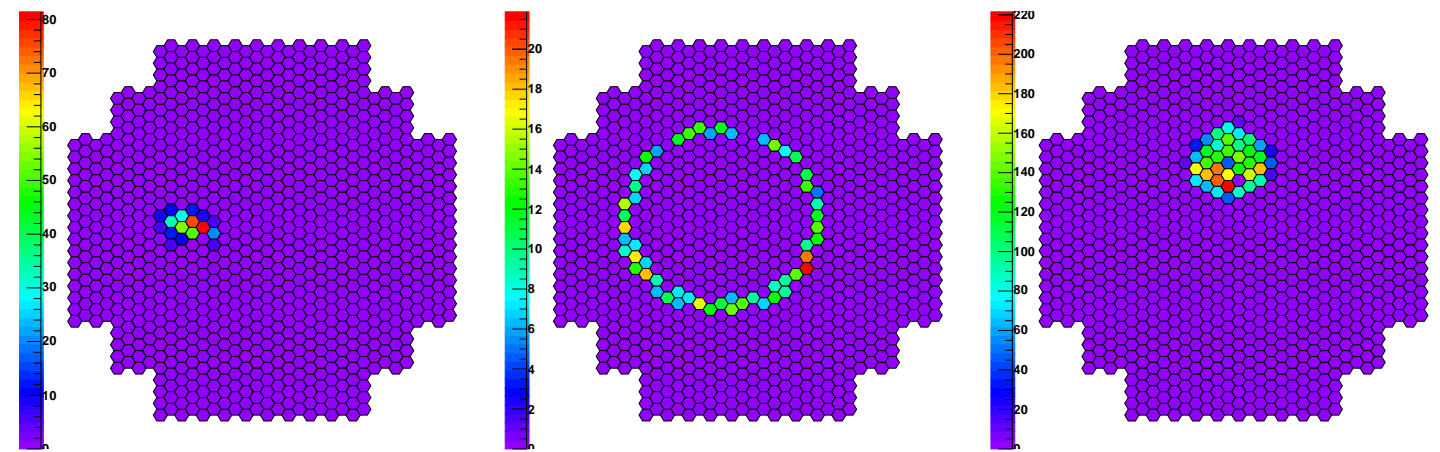

Figure 1: Example event displays for the different types of measured events. Left: Cosmic event; Middle: Muon ring; Right: UAV event. The colour scale indicates the number of produced photo-electrons. Each pixel has a field-of-view of $0.16^{\circ}$ diameter.

\section{Reconstruction and analysis of UAV events}

As a first step, the standard calibration and data cleaning procedures were applied to all recorded events: First, the standard pedestal subtraction and gain calibration procedure were applied [9]. Then, non-operational pixels were interpolated using the mean intensity of the neighbouring operational pixels to estimate the intensity for the non-operational pixel. Finally, the data were cleaned, retaining only pixels fulfilling a dual-threshold condition: the pixel has recorded at least seven photo-electrons and a neighbouring pixel at least five photo-electrons, or vice-versa [10].

After calibration and cleaning, the UAV events need to be identified among the background events. As illustrated in Figure 1, there are three types of events in the data: (1) cosmic events, i.e., events from high energy electrons, hadrons or photons entering the atmosphere charcterised by an elongated elliptical or irregular shape; (2) muon rings, i.e., Cherenkov rings produced by atmospheric muons (also originating from comic events) crossing the telescope and characterised by their ring form, and (3) UAV events characterised by their regular hexagonal shape. As the bulk of the Cherenkov emission in the H.E.S.S. sensitive energy range is produced at about $8 \mathrm{~km}$ altitude above the H.E.S.S. array and the telescopes are in convergent pointing towards a position at about $200 \mathrm{~m}$ altitude, it is very unlikely for cosmic events (and for muon rings even more) to be recorded in three or four telescopes, and so they are recorded in one or two telescopes. UAV events, on the other hand, are recorded in four telescopes except if telescopes are unavailable due to dead-time. Because of this, after events which were not completely included in the camera have been removed by applying a cut of $0.034 \mathrm{rad}\left(\approx 2^{\circ}\right)$ on the nominal distance (i.e., the angular distance between the centre of the camera and the image centre of gravity (COG)), events recorded in at least three telescopes were selected as UAV events. The performance of this cut was verified by setting up the UAV such that UAV events have a higher image amplitude than most cosmic events and checking that the timing distribution of the selected events agrees with the frequency of the UAV-mounted LED. No event classified wrongly as UAV event could be found using both of these methods.

For run A, 343 events out of a run total of 41000 where selected as UAV events, while for run B, 350 out of 27000 were selected.

The UAV moves in the field of view due drift in the GNSS navigation and buffeting from atmospheric turbulence. This makes precise tracking of the UAV necessary in order to get its 
position and hence its distance to the different telescopes, which is a critical quantity for the crosscalibration. The UAV position was obtained by triangulation using the images of the light source on the camera (the event displays): the COG of the image corresponds to a direction in the field of view (with respect to the known pointing direction) and the most likely position of the UAV is taken to be the analytically determined value which minimizes the sum of squared distances to the lines of sight reconstructed for the individual telescopes. We found that the UAV moved up to $30 \mathrm{~m}$ in vertical direction and less than $5 \mathrm{~m}$ in horizontal direction after having entered the field of view of the telescopes.

By subdividing the duration during which the UAV was visible to at least three telescopes ( $400 \mathrm{~s}$ to $500 \mathrm{~s}$ ) in bins of $5 \mathrm{~s}$ during which the intrinsic movement of the UAV is excepted to be small (except while the UAV was moving in and out of the field of view), the position distribution within bins follows a Gaussian distribution. Adding the distributions after centering them on the same value, leads to a statistical position uncertainty of $5 \mathrm{~cm}$ perpendicular to the pointing direction of the telescopes and $50 \mathrm{~cm}$ along the pointing direction. By comparing the positions reconstructed with this method to the positions obtained from the independent UAV GNSS receiver, systematic uncertainties were estimated to be at least $5 \mathrm{~cm}$ perpendicular to pointing and $1.1 \mathrm{~m}$ along the pointing direction respectively and at most $8 \mathrm{~m}$ for both pointing directions. As $8 \mathrm{~m}$ is within the expected accuracy of the GNSS, it is unclear from which of both position determination methods an $8 \mathrm{~m}$ offset seen between both methods comes from, and hence this big range for the systematic uncertainty of the reconstructed position.

From simple geometric considerations, one would expect that:

$$
I \propto \frac{1}{d^{2}}+O\left(\frac{1}{d^{4}}\right)
$$

where $I$ is the sum of all the photo-electrons in an event, in a given telescope and $d$ the distance of the UAV to the mirror plane of this telescope. This has been verified with a Monte-Carlo simulation. It turned out that this proportionality holds modulo point-to-point variations of about $1 \%$ and a decrease with the amount of matter the photons pass due to atmospheric absorption. This decrease leads to differences of about $1 \%$ in the expected intensity for the different telescopes for the configuration used in this campaign. This then allows the specific relative efficiencies $\epsilon_{i}$ of the telescopes $i$ to be defined for each four-telescope event as:

$$
\epsilon_{i}=\frac{\left(I \times d^{2} \times C\right)_{i}}{\left\langle\left(I \times d^{2} \times C\right)_{j}\right\rangle}
$$

where $I$ and $d$ are defined as before, $C$ is a minor correction factor close to 1 encompassing the $O\left(\frac{1}{d^{4}}\right)$ from equation (1) and the atmospheric absorption and $\left\langle\left(I \times d^{2} \times C\right)_{j}\right\rangle$ is the average of $I \times d^{2} \times C$ over all telescopes. The point-to-point variations cannot be corrected for as they occur on too small a scale. The run-wise relative efficiencies are then just the averaged event-wise relative efficiencies.

The position of the UAV was determined by minimizing the sum of the squared distances of the UAV to the line of sight towards the UAV obtained from each telescope using the COG of the image. The best fit position is not exactly on the determined lines of sight and so there are remaining residuals on the COG which are, among other, due to slight mispointings of the telescopes leading to an incorrect reconstructed direction. This allows us to estimate the mispointings of the telescopes. 

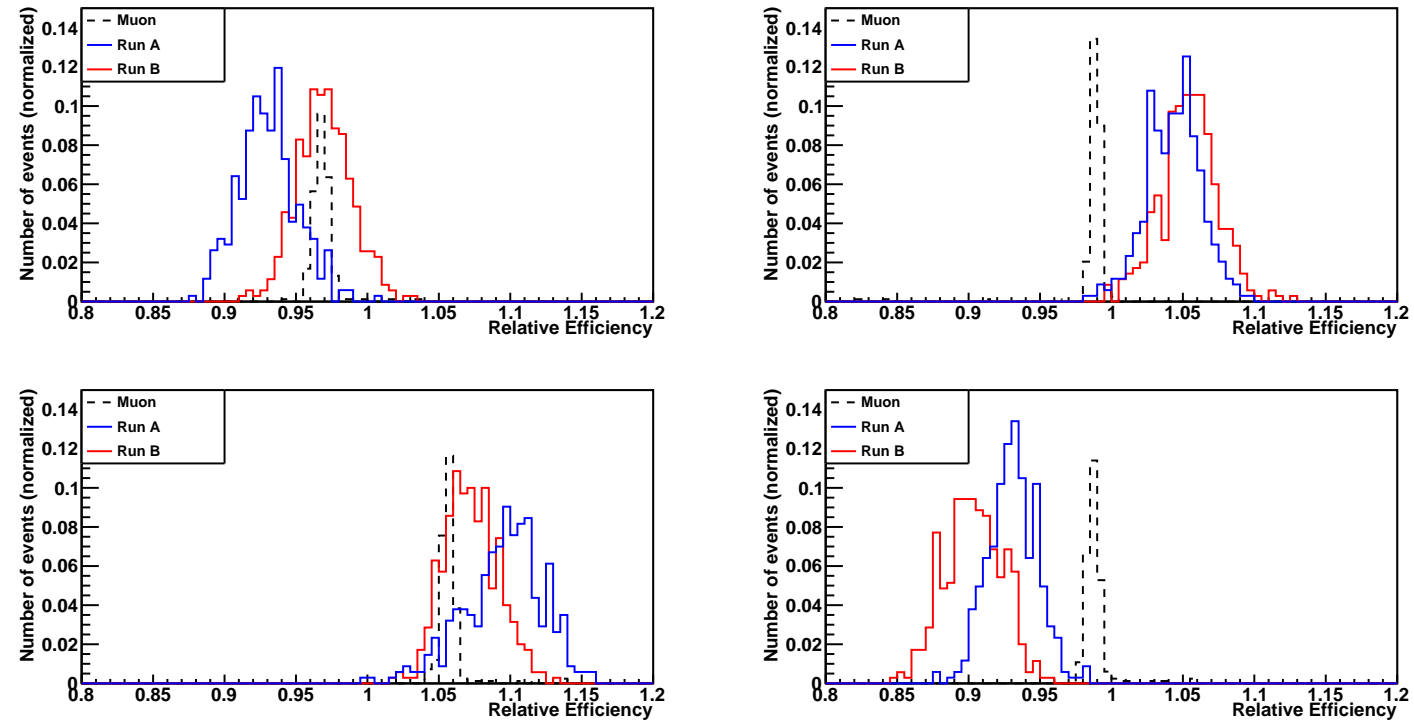

Figure 2: Normalized distribution of the relative efficiencies on an event-by-event basis as determined with the UAV for run A (blue) and run B (red) for all 4 HESS-I telescopes. In addition, the distribution of the relative muon efficiencies over the whole observation period on a run-by-run basis is shown in dashed black. It has been rescaled to the height of the other distributions for easy comparison.

\section{Results \& Discussion}

\subsection{Cross-Calibration}

The relative efficiency distributions obtained for each telescope in the two UAV runs, considering only events which were UAV-like in all four telescopes, are shown in Figure 2. Even though the distributions are not fully consistent between the two runs, the average relative efficiency in the individual telescopes deviates by only $3.1 \%$. This shows that these two runs, which were conducted on different nights with different observing and environmental conditions, lead to relative telescope efficiencies, which are consistent within this margin of error. The difference between the average relative efficiency of both runs could be due to a number of phenomena: broken pixels for which the intensity is not exactly recovered during interpolation, systematic uncertainties in the position determination leading to an imprecise correction of the expected intensity, the point-to-point variations of about $1 \%$ and, on a smaller scale, the uncertainties in the flat-fielding and the difference between the wavelengths of the flat-fielding LEDs $(370 \mathrm{~nm})$ and of the UAV-mounted light source $(400 \mathrm{~nm})$. Investigating these factors in depth requires more UAV calibration runs to be performed.

The optical efficiency calibration of the H.E.S.S. telescopes is usually performed with atmospheric muons. It is not expected to have any common systematic uncertainties with UAV calibration, beside atmospheric absorption and the uncertainties intrinsic to any calibration procedure using a light source outside of the telescopes, as both calibration methods are based on completely different processes. The main operational uncertainties are the uncertainty on the pedestals and the uncertainty on the flat-fielding, however both of these uncertainties take values far below the statistical uncertainty from the width of the distributions in Figure 2. The atmospheric 

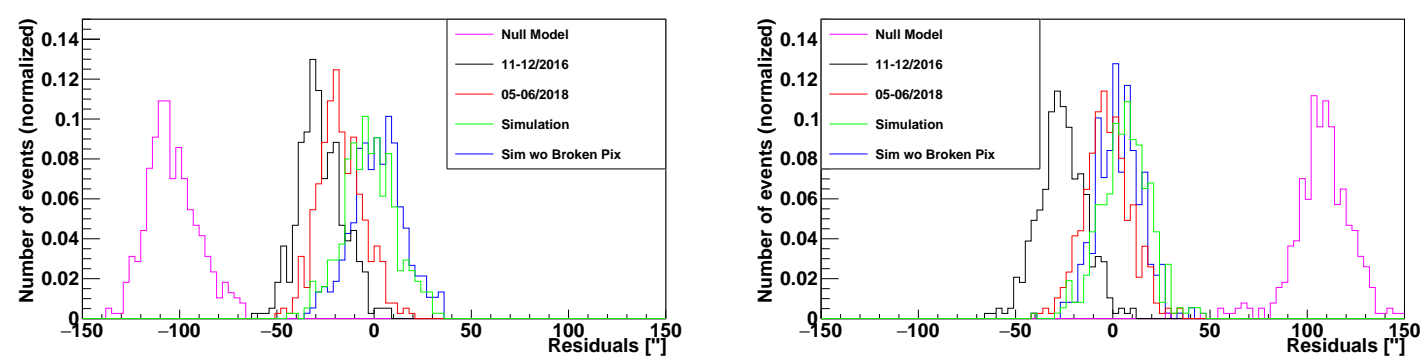

Figure 3: Distribution of the residuals on the COG for the different pointing models for one telescope and one of the two camera coordinates per run ( $\mathrm{x}$ and $\mathrm{y}$ axis). The shown telescope and camera coordinate were chosen in a way that the residual distribution corresponds the most to the average distribution for the model and run (as they were looking quite differently for the different telescopes and runs). Left: Residuals on X-coordinate of COG in CT2 for run A. Right: Residuals on X-coordinate of COG in CT3 for run B. Null Model: Model without any pointing corrections; 11-12/2016: H.E.S.S. standard pointing model based on data from November and December 2016; 05-06/2018: H.E.S.S. standard pointing model based on data from May and June 2018 (i.e. taken around the measurement period), except for CT4 where data from December 2017 and January 2018 was used; Simulation: Residuals obtained from simulation using broken pixels detected in data of runs and perfect pointing; Sim wo Broken Pix: Residuals obtained from simulation without broken pixels and perfect pointing.

absorption, on the other hand, leads to corrections of about $0.5 \%$ for the UAV cross-calibration. It is difficult to qualify the uncertainty of this value, given the limited number of measurements available under Namibian atmospheric conditions and this so needs further investigations during which the UAV is moved to different positions. However, as the muon optical efficiency calibration is based on data from multiple very different pointings, the effect of the atmospheric absorption on the computed average relative efficiencies is expected to average out over this different pointings and so this should not be a common uncertainty for the relative efficiencies. As there are no other common uncertainties, the muon calibration method is well suited for a cross-check of the UAV cross-calibration method and, for this reason, the distribution of the relative efficiencies from this method is also shown in Figure 2, on a run-by-run basis for the whole observation period (25 nights) due to the run-by-run variations of the muon optical efficiencies. The deviation between this relative efficiency and that obtained with the UAV is $5.5 \%$ and $6.3 \%$ respectively for the two runs. As both methods are not expected to have any systematics in common except for the atmospheric absorption and telescope operational uncertainties, this consistency for the different runs is a strong indication that the uncertainties of both methods are of the same order of magnitude or less (if one of the methods performs substantially better than the other one). The size of the systematic uncertainties of the UAV calibration method will be further constrained by comparing its results to further independent methods such as the air shower optical efficiency calibration method [11].

\subsection{Pointing corrections}

The distribution of the residuals on the COG compared for three pointing correction models used on the taken data and two Monte-Carlo simulations are shown in Figure 3, selecting one telescope and coordinate for each run for illustration purposes such that the residual distribution means correspond best to the average distribution means over the 4 telescopes and the 2 coordinates 
( $\mathrm{x} \& \mathrm{y})$. The three pointing models used on the taken data are first the so-called Null Model (i.e. one assumes that there is no structure deformation leading to mispointings and that the nominal pointing corresponds to the actual pointing) and two models obtained with the H.E.S.S. standard procedure for creating pointing correction models, one constructed from data taken in November and December 2016 (the latest model available at the moment of data taking and one and half a year old at this time) and the other one from data taken in May and June 2018, covering the period where the UAV runs were carried out, except for CT4 where no data from this period is available due to a hardware failure (therefore the last available pointing model from December 2017 and January 2018 was used for CT4). One recovers the expected result that the Null Model performs the worst and that the up-to-date pointing model performs the best, and so the UAV data can provide an additional verification for the derived pointing models.

This shows that UAV events can be used as an additional method to investigate the accuracy of a telescope's pointing model, but leaves the question open whether it is possible to ameliorate the pointing corrections with UAV events. For this reason, Monte-Carlo simulations using perfect pointing, and accounting for as many other physical phenomena as possible, were run to disentangle the part of the residuals due to mispointings from the part due to other operational considerations, once using the non-operational pixels as determined in the simulated UAV run and once with all pixels operational. The residuals have a similar size using the up-to-date pointing model on the data and for the simulation with broken pixels. This indicates that the standard pointing corrections already reach the maximum precision achievable with the UAV with the current data analysis method. However, there is an improvement between the simulation with and without broken pixels, which shows that not only mispointings lead to a shift in the residuals, but that broken pixels shift them too, and so that going beyond a simple interpolation to recover broken pixels could possibly allow to disentangle the shift due to broken pixels from the one due to mispointings.

The residuals found with the up-to-date pointing model have a size of about $10^{\prime \prime}$. This is similar to the uncertainty on the source position determination with H.E.S.S. due to systematic pointing uncertainties found in a previous study [12]. This shows that the UAV already achieves similar accuracy, even without implementing more elaborate methods to recover broken pixels.

\section{Conclusion}

Here, we present the results of the first cross-calibration of an IACT array with a UAV-based light source. The obtained cross-calibration was consistent within $5.5 \%$ and $6.3 \%$ respectively with the muon cross-calibration for the two runs taken. As these two cross-calibration methods are based on very different processes, they are not expected to have any common systematic uncertainties besides those intrinsic to a calibration method based on a light source at a distance hundreds of meters from the telescopes which have been discussed previously. As they are on a smaller scale, this is a strong indication of both methods having uncertainties of this order of magnitude or less. This means that UAVs are well suited to cross-calibrate Cherenkov telescope arrays and that crosscalibration with a single light source on an event-by-event basis are indeed possible. This result has been achieved in a first attempt with a non-optimised first-generation UAV prototype and so the uncertainty will be improved through a better understanding of the systematic uncertainties of the 
technique, as well as further iterations of the UAV prototype with a custom-made UAV platform, improvements to the light source and a better integration of the calibration payload to the UAV [6].

We have also shown that we can, without any additional data, verify the pointing corrections of the H.E.S.S. telescopes by comparing the effect that different telescope pointing models have on the observed UAV data.

Finally, the UAV also allows other calibration issues to be tackled. The UAV permits - unlike the muon cross-calibration - a multiwavelength cross-calibration to be performed by just changing the LED and thus allowing the wavelength dependent effects to be monitored. It is also possible to monitor the transparency of the lowest layers of the atmosphere with the UAV - either by integrating meteorological devices in the calibration payload (as proposed in [6]) or by trying to infer the atmospheric absorption from the amount of light recorded in the different telescopes.

\section{Acknowledgements}

We thank Prof. S. Wagner, director of the H.E.S.S. Collaboration and Prof. O. Reimer, chairman of the H.E.S.S. Collaboration board, for allowing us to use H.E.S.S. data in this publication. AMB acknowledges the financial support of the Royal Society Research Grant RG160883 that funded the construction of the prototype used in this campaign, and funded the logistics associated with the campaign. We would also like to thank Matthias Buchele and David Jankowsky, the on-site shiftcrew during the UAV campaign, for their help in setting up the H.E.S.S. array without whom it would not have been possible to take any data. Alison Mitchell and Vincent Marandon for their help with the muon calibration. And Jean-Philippe Lenain for helping us with the H.E.S.S. detector simulation. Finally, our thanks go to all the members of the H.E.S.S. Collaboration for their technical support and for many stimulating discussions which undoubtedly improved the quality of this contribution.

\section{References}

[1] B. S. Acharya et al., Astropart. Phys. 43 (2013) 3

[2] J. N. Matthews, Progress Towards a Cross-Calibration of the Auger and Telescope Array Fluorescence Telescopes via an Air-borne Light Source, ICRC (2013)

[3] M. Hayashi et al., Pos ICRC2015 (2016) 692

[4] C. Chang et al., PASP 127 (2015) 1131

[5] A. G. Basden et al., MNRAS 477 (2018) 2209

[6] A. M. Brown, Astropart. Phys. 97 (2018) 69

[7] A. M. Brown et al., Pos ICRC2015 (2016) 934

[8] R. Cornils et al., Astropart. Phys. 20 (2003) 119

[9] F. Aharonian et al., Astropart. Phys. 22 (2004) 109

[10] F. Aharonian et al., A\&A 457 (2006) 899

[11] A. M. W. Mitchell et al., Astropart. Phys. 75 (2016) 1

[12] S. Gillessen, Ph.D. thesis, Ruprecht-Karls-Universität Heidelberg (2004) 